Case Reports in
Gastroenterology
Case Rep Gastroenterol 2020;14:547-553

DOI: 10.1159/000508929

Published online: October 28, 2020

(C) 2020 The Author(s)

Published by S. Karger AG, Base www.karger.com/crg

This article is licensed under the Creative Commons Attribution-NonCommercial 4.0 International License (CC BY-NC) (http://www.karger.com/Services/OpenAccessLicense). Usage and distribution for commercial purposes requires written permission.

\title{
STK11 p.F354L Germline Mutation in a Case of Multiple Gastrointestinal Tumors
}

\author{
Yohei Kojima $^{a} \quad K^{2}$ ouki Ohtsuka ${ }^{b}$ Shun Ishii ${ }^{a}$ Nobuyoshi Aso ${ }^{a}$ \\ Atsuko Ohki ${ }^{a}$ Yoshikazu Hashimoto ${ }^{a}$ Hirohisa Takeuchia \\ Hiroaki Ohnishib Nobutsugu Abe ${ }^{a}$

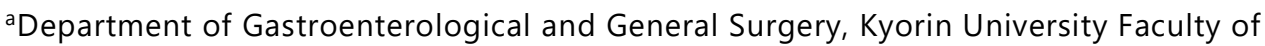 \\ Medicine, Tokyo, Japan; ${ }^{b}$ Department of Laboratory Medicine, Kyorin University Faculty of \\ Medicine, Tokyo, Japan
}

\section{Keywords}

STK11 · p.F354L · Duodenal adenoma · Gastric adenoma $\cdot$ Colorectal adenocarcinoma $\cdot$ Liver metastasis

\begin{abstract}
Serine/threonine kinase 11 (STK11) is known as a critical tumor-suppressor gene that is frequently mutated in a broad spectrum of human cancers. Among these, the p.F354L mutation of STK 11 has been identified in sporadic colon or lung cancer cases. Here, we report the case of a 75-year-old male patient who underwent surgical treatment for multiple tumors of the gastrointestinal system. Genetic mutations were screened in all resected samples, including duodenal high-grade adenoma, gastric high-grade adenoma, rectal adenocarcinoma, and liver metastasis of rectal adenocarcinoma, by next-generation sequencing for mutational hotspots involving 50 oncogenes and tumor suppressor genes. The characteristic hamartomatous polyp of Peutz-Jeghers syndrome was not detected in any tumor specimen. However, all samples as well as the normal rectal mucosa harbored the genetic mutation p.F354L in STK11. In addition, somatic mutations coexisted in the tumor samples, including KRAS p.A146T, TP53 p.G238X, and APC p.T1556fs in the duodenal adenoma; TP53 p.G238Y and APC p.T1556fs in the gastric adenoma; and TP53 p.R282W in the rectal adenocarcinoma and metastatic liver cancer. No somatic mutation was detected in the normal rectal mucosa as a control sample. To our
\end{abstract}




\section{Case Reports in Gastroenterology}

Case Rep Gastroenterol 2020;14:547-553

DOI: $10.1159 / 000508929$

(c) 2020 The Author(s). Published by S. Karger AG, Basel www.karger.com/crg

Kojima et al.: STK11 p.F354L Mutation in Multiple Gastrointestinal Cancer

knowledge, this is the first report of an STK11 germline mutation in a patient with multiple tumors of the gastrointestinal tract.

\section{Introduction}

Mutation in serine/threonine kinase 11 (STK11), also known as liver kinase B1 (LKB1), was first identified as the causal mutation in Peutz-Jeghers syndrome (PJS), a rare inherited autosomal dominant disorder characterized by the development of benign gastrointestinal hamartomas and the early onset of cancer [1]. Since then, STK11 has become recognized as a critical tumor-suppressor gene that is frequently mutated in a broad spectrum of human cancers.

STK11 directly phosphorylates and regulates adenosine monophosphate-activated protein kinase [2, 3]. In particular, the STK11 p.F354L mutation has been reported in cases of sporadic colon cancer [4]. We also reported that the STK11 p.F354L mutation may play a key role in the development of duodenal adenoma and adenocarcinoma [5].

Here, we report the first case of a patient with multiple tumors (stomach, duodenum, and rectum) of the gastrointestinal tract harboring an STK11 germline mutation without specific PJS findings.

\section{Case Report}

A 75-year-old man presented to another hospital with anemia. Gastrointestinal endoscopies revealed gastric, duodenal, and rectal tumors, and the patient was referred to our hospital for treatment. The patient had not been diagnosed with PJS previously. His family members did not have any signs or symptoms related to PJS. Esophagogastroduodenoscopy demonstrated a protruding tumor (12 $\mathrm{mm}$ in diameter) on the lesser curvature side of the gastric body (Fig. 1b) and another protruding tumor $(20 \mathrm{~mm}$ in diameter) in the duodenal bulb (Fig. 1a). Endoscopic punch biopsies of the tumors revealed a gastric high-grade adenoma and a duodenal high-grade adenoma, respectively. Furthermore, colorectal endoscopy revealed an ulcerated tumor in the rectum (Fig. 1c), and endoscopic punch biopsy revealed an adenocarcinoma. An abdominal CT (computed tomography) scan revealed neither regional lymph node swelling nor lung/liver metastases.

The gastric and duodenal adenomas were resected by endoscopic submucosal resection. The rectal cancer was resected by laparoscopic high-anterior resection. Histopathologically, the duodenal (Fig. 3a) and gastric (Fig. 3b) tumors were both graded as high-grade dysplasia adenoma based on the World Health Organization tumor classification system according to differences in the atypical ductal structure, nuclear atypia, and cytoplasm/nucleus ratio. The rectal tumor was histologically diagnosed as a well-differentiated adenocarcinoma (Fig. 3c). This tumor invaded the rectal muscularis propria with fibrosis. Moderate lymphatic and venous invasion were observed. One lymph node metastasis was diagnosed. According to the TNM staging system, the rectal cancer stage was IIIA. Histologically, these tumors (stomach, duodenum, and rectum) exhibited no characteristic feature of the hamartomatous polyp of PJS.

A hepatic tumor was detected at the 1-year follow-up by enhanced CT scanning (Fig. 2). The lesion was found in segment 8 of the hepatic area with a size of $3 \mathrm{~cm}$, and was surgically resected. Histologically, the hepatic tumor was diagnosed as a metastatic adenocarcinoma 


\section{Case Reports in Gastroenterology}

Case Rep Gastroenterol 2020;14:547-553

DOI: $10.1159 / 000508929$

(c) 2020 The Author(s). Published by S. Karger AG, Basel www.karger.com/crg

Kojima et al.: STK11 p.F354L Mutation in Multiple Gastrointestinal Cancer

from rectal cancer (Fig. 3d). This patient has had no recurrence for more than 5 years after the hepatic surgery without any chemotherapy.

Subsequently, we carried out molecular analyses by next-generation sequencing [5] to screen for genetic mutations in the tumor samples and a normal rectal mucosa sample as the control (Fig. 3e). Genomic DNA was extracted from formalin-fixed paraffin-embedded sections $(10 \mu \mathrm{m})$ of all samples using the ZR FFPE DNA Miniprep kit (Zymo Research, Irvine, CA, USA) in accordance with the manufacturer's protocol. DNA integrity was examined by amplification of control genes using real-time PCR (polymerase chain reaction) with the QuantStudio 6 Flex Real-Time PCR system (Thermo Fisher Scientific), producing fragments of approximately $200 \mathrm{bp}[6,7]$. Samples with DNA integrity equal to or higher than 150 pmol were selected for analysis of mutational hotspots by next-generation sequencing.

To analyze the mutational hotspots of 50 oncogenes and tumor suppressor genes, we used the Ion AmpliSeq Cancer Hotspot Panel v2 (Life Technologies, Carlsbad, CA, USA) on an Ion Torrent Personal Genome machine (Ion PGM; Life Technologies); the genes analyzed were ABL1, AKT1, ALK, APC, ATM, BRAF, CDH1, CDKN2A, CSF1R, CTNNB1, EGFR, ERBB2, ERBB4, EZH2, FBXW7, FGFR1, FGFR2, FGFR3, FLT3, GNA11, GNAS, GNAQ, HNF1A, HRAS, IDH1, JAK2, JAK3, IDH2, KDR, KIT, KRAS, MET, MLH1, MPL, NOTCH1, NPM1, NRAS, PDGFRA, PIK3CA, PTEN, PTPN11, RB1, RET, SMAD4, SMARCB1, SMO, SRC, STK11, TP53, and VHL. We performed sequencing on a 318 chip using a Sequencing kit 200 v2 (Thermo Fisher Scientific) according to the manufacturer's protocols. Signal processing, mapping, and quality control were performed with Torrent Suite v4.2 (Thermo Fisher Scientific). Sequence variants were called using Ion Reporter v4.2 (Thermo Fisher Scientific) and the AmpliSeq CHPv2 single-sample workflow with default settings. Variants were subsequently filtered to include only exonic, nonsynonymous variants with allelic frequencies higher than $10 \%$. A sequence variation was considered to be a potential mutation when the coverage was above $100 \times$ and the allelic frequency was above $10 \%$. The samples were examined using SIFT and Polymorphism Phenotyping v2 (PolyPhen-2); a SIFT score of $<0.05$ indicates that the substitution is carcinogenic, and PolyPhen-2 scores in the range of $0.15-0.85$ suggest that the substitution is carcinogenic, while scores of $0.85-1.0$ indicate that the substitution is likely carcinogenic $[8,9]$.

All samples harbored the same mutation: STK11 p.F354L. Somatic mutations coexisted in the tumor samples, including KRAS p.A146T, TP53 p.G238X, and APC p.T1556fs in the duodenal adenoma, and TP53 p.G238Y and APC p.T1556fs in the gastric adenoma. Therefore, the same APC and TP53 mutations were detected in the duodenal adenoma and gastric adenoma. The rectal adenocarcinoma and liver metastasis had the common somatic mutation TP53 p.R282W. The rectal mucosa harbored only the mutation STK11 p.F354L. No somatic mutation was detected in the normal rectal mucosa as the control sample.

Moreover, microsatellite instability (MSI) was only tested in the duodenal high-grade adenoma sample. The MSI status was assessed within common microsatellite locations (BAT25, BAT26, NR21, NR24, and NR27), which revealed no deficient mismatch repair phenotype.

\section{Discussion}

The germline mutation STK11 p.F354L was detected in a case of multiple tumors (gastric high-grade dysplasia adenoma, duodenal high-grade dysplasia adenoma, and rectal adenocarcinoma) of the gastrointestinal tract by next-generation sequencing-based screening of more than 2,800 mutational hotspots among 50 oncogenes and tumor suppressor genes [5]. Nextgeneration sequencing revealed the STK11 p.F354L mutation in all samples (including 


\section{Case Reports in Gastroenterology}

Case Rep Gastroenterol 2020;14:547-553

DOI: $10.1159 / 000508929$

c) 2020 The Author(s). Published by S. Karger AG, Basel www.karger.com/crg

Kojima et al.: STK11 p.F354L Mutation in Multiple Gastrointestinal Cancer

metastatic adenocarcinoma of the rectal cancer) coexisting with somatic mutations, including the control sample.

The point mutation STK11 p.F354L has previously been reported in cases of lung cancer or PJS [10-13]. Our patient had no familial history or typical characteristics and symptoms of PJS. Therefore, this is the first report of a patient with multiple primary tumors, including adenoma, associated with this STK11 mutation. Moreover, this is the first report of a comprehensive screening effort with next-generation sequencing in a case of multiple tumors, including high-grade dysplasia adenoma, revealing an STK11 germline mutation.

In vitro analyses showed that STK11 with the p.F354L mutation activates AMPK-like kinases, thereby enhancing the proliferation of cells transfected with these mutations [10-12]. Notably, p.F354L was recently reported as a single nucleotide polymorphism prevalent in the Japanese population at frequencies of 1-3\% (UCSC Genome Bioinformatics). Moreover, the ClinVar database from the National Library of Medicine classifies this point of mutation as benign/likely benign. However, we previously reported that STK11 mutation was confirmed in $25 \%$ of duodenal adenocarcinomas and $9 \%$ of duodenal adenomas, which are higher rates than the reported prevalence in the Japanese population [5]. Therefore, our study suggests that the STK11 p.F354L mutation should be further validated considering its potential key role in the development of duodenal adenoma and adenocarcinoma.

Schabath et al. [14] reported the presence of commonly co-occurring mutations in the STK11 or TP53 tumor suppressor genes in lung cancer, which were suggested to confer tumorigenic ability in KRAS-mutant tumors. Mutation in TP53 was strongly associated with enhanced proliferation, and STK11 mutation was associated with the suppression of immune surveillance [14]. The same mechanisms of tumorigenicity may also be involved in gastrointestinal tumors, although this hypothesis requires further direct testing [5]. We also detected somatic mutations coexisting in the same tumor. KRAS, TP53, and APC mutations were detected in the duodenal adenoma, and the TP53 p.R282W mutation was detected in both the rectal adenocarcinoma and metastatic liver cancer. Therefore, these combinations of genetic mutations may affect tumorigenesis not only in the lung but also in the gastrointestinal tract.

One of the key findings of our case was that there was the same STK11 mutation in multiple gastrointestinal tract tumors. Hemminki et al. [1] suggested the possibility that STK11 may not only be a tumor suppressor gene that regulates hamartoma formation but might also play an important role as an early gatekeeper in the hamartoma-adenoma-carcinoma sequence. However, we did not detect a hamartoma in the gastrointestinal tract in the present case; therefore, validating this hypothesis requires further accumulation of cases associated with STK11 mutation.

MSI may also be present in these tumors and could play important roles in their development [6]. However, MSI was only tested in the duodenal high-grade adenoma sample and was negative. Therefore, MSI is considered to be very unlikely to have played a role in the tumorigenesis of this case.

In summary, we reported an unusual case of multiple tumors of the gastrointestinal tract associated with an STK11 germline mutation. Based on comprehensive molecular analysis, we suggest that the germline mutation of STK11 p.F354L may cause gastrointestinal tumors, including adenoma, and we also found coexisting somatic mutations of TP53, APC, and KRAS in the tumors of this patient. This case suggests that in a patient with STK11 mutations without symptoms of PJS, the carcinogenesis pathway may involve development of adenocarcinomas due to malignant transformation from adenoma. Further functional studies are needed to establish the precise role of STK11 in cancer development of the gastrointestinal tract. 


\section{Case Reports in Gastroenterology}

\begin{tabular}{l|l}
\hline DOI: 10.1159/000508929 & (c) 2020 The Author(s). Published by S. Karger AG, Basel \\
\hline
\end{tabular} www.karger.com/crg

Kojima et al.: STK11 p.F354L Mutation in Multiple Gastrointestinal Cancer

\section{Statement of Ethics}

This study was approved by the Research Ethics Committee of Kyorin University Faculty of Medicine. Informed consent was obtained from the patient.

\section{Conflict of Interest Statement}

The authors have no conflicts of interest to declare.

\section{Funding Sources}

No source of funding was received for this work.

\section{Author Contributions}

Y. Kojima proposed the concept and wrote the manuscript. K. Ohtsuka provided technical support and advice. H. Ohnishi supervised the laboratory work. S. Ishii, N. Aso, A. Ohki, Y. Hashimoto, and H. Takeuchi provided advice. N. Abe led the surgery and study design and drafted the manuscript.

\section{References}

1 Hemminki A, Markie D, Tomlinson I, Avizienyte E, Roth S, Loukola A, et al. A serine/threonine kinase gene defective in Peutz-Jeghers syndrome. Nature. 1998 Jan;391(6663):184-7.

2 Shackelford DB, Shaw RJ. The LKB1-AMPK pathway: metabolism and growth control in tumour suppression. Nat Rev Cancer. 2009 Aug;9(8):563-75.

3 Islam MI, Khan AM, Parves MR, Hossain MN, Halim MA. Prediction of deleterious non-synonymous SNPs of human STK11 gene by combining algorithms, molecular docking, and molecular dynamics simulation. Sci Rep. 2019 Nov;9(1):16426.

4 Forcet C, Etienne-Manneville S, Gaude H, Fournier L, Debilly S, Salmi M, et al. Functional analysis of PeutzJeghers mutations reveals that the LKB1 C-terminal region exerts a crucial role in regulating both the AMPK pathway and the cell polarity. Hum Mol Genet. 2005 May;14(10):1283-92.

5 Kojima Y, Ohtsuka K, Ohnishi H, Abe N, Furuse J, Watanabe T, et al. APC:T1556fs and STK11 mutations in duodenal adenomas and adenocarcinomas. Surg Today. 2018 Aug;48(8):765-72.

6 Laforest A, Aparicio T, Zaanan A, Silva FP, Didelot A, Desbeaux A, et al. ERBB2 gene as a potential therapeutic target in small bowel adenocarcinoma. Eur J Cancer. 2014 Jul;50(10):1740-6.

7 Chlumská A, Waloschek T, Mukenšnabl P, Martínek P, Kašpírková J, Zámečník M. Pyloric gland adenoma: a histologic, immunohistochemical and molecular genetic study of 23 cases. Cesk Patol. 2015;51(3):137-43.

8 Kumar P, Henikoff S, Ng PC. Predicting the effects of coding non-synonymous variants on protein function using the SIFT algorithm. Nat Protoc. 2009;4(7):1073-81.

9 Adzhubei IA, Schmidt S, Peshkin L, Ramensky VE, Gerasimova A, Bork P, et al. A method and server for predicting damaging missense mutations. Nat Methods. 2010 Apr;7(4):248-9.

10 Buryk MA, Picarsic JL, Creary SE, Shaw PH, Simons JP, Deutsch M, et al. Identification of unique, heterozygous germline mutation, STK11 (p.F354L), in a child with an encapsulated follicular variant of papillary thyroid carcinoma within six months of completing treatment for neuroblastoma. Pediatr Dev Pathol. 2015 Jul-Aug;18(4):318-23.

11 Suzuki Y, Oonishi T, Kudo T, Doi H. LKB1, TP16, EGFR, and KRAS somatic mutations in lung adenocarcinomas from a Chiba Prefecture, Japan cohort. Drug Discov Ther. 2012 Feb;6(1):24-30.

12 Grünewald I, Vollbrecht C, Meinrath J, Meyer MF, Heukamp LC, Drebber U, et al. Targeted next generation sequencing of parotid gland cancer uncovers genetic heterogeneity. Oncotarget. 2015 Jul;6(20):18224-37. 


\section{Case Reports in Gastroenterology}

\begin{tabular}{l|l}
\hline Case Rep Gastroenterol 2020;14:547-553 \\
\hline DOI: 10.1159/000508929 & $\begin{array}{l}\text { @ 2020 The Author(s). Published by S. Karger AG, Basel } \\
\text { www.karger.com/crg }\end{array}$ \\
\hline
\end{tabular}

Kojima et al.: STK11 p.F354L Mutation in Multiple Gastrointestinal Cancer

13 Rousset-Jablonski C, Gompel A. Screening for familial cancer risk: focus on breast cancer. Maturitas. 2017 Nov;105:69-77.

14 Schabath MB, Welsh EA, Fulp WJ, Chen L, Teer JK, Thompson ZJ, et al. Differential association of STK11 and TP53 with KRAS mutation-associated gene expression, proliferation and immune surveillance in lung adenocarcinoma. Oncogene. 2016 Jun;35(24):3209-16.
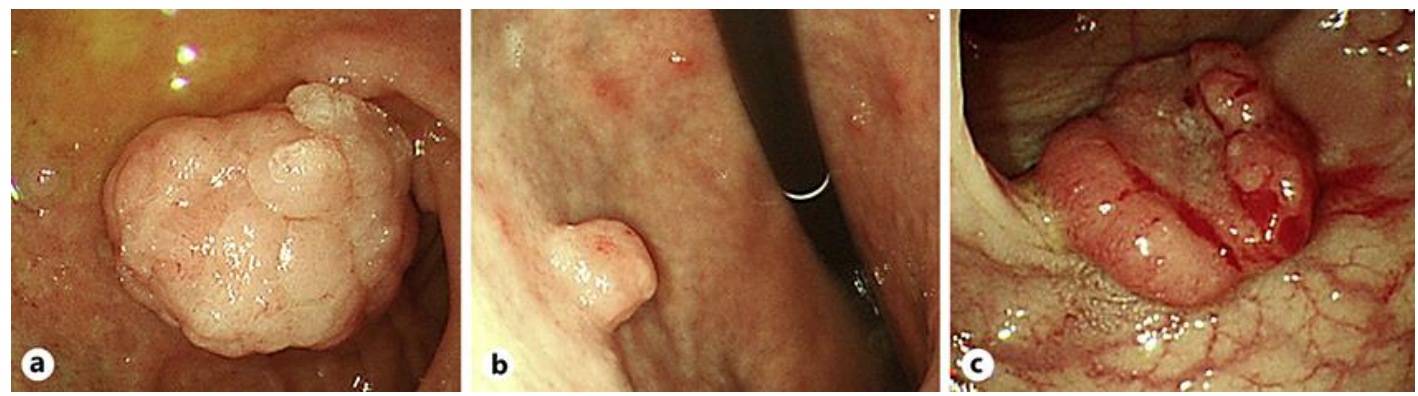

Fig. 1. Endoscopic findings. a Duodenal polyp $(2 \mathrm{~cm})$ on the bulb portion with a protruded shape. b Gastric polyp $(1.2 \mathrm{~cm})$ with a protruded shape on the anterior wall of the gastric body. $\mathbf{c}$ Rectal adenocarcinoma $(5 \mathrm{~cm})$ with an ulcerative region.

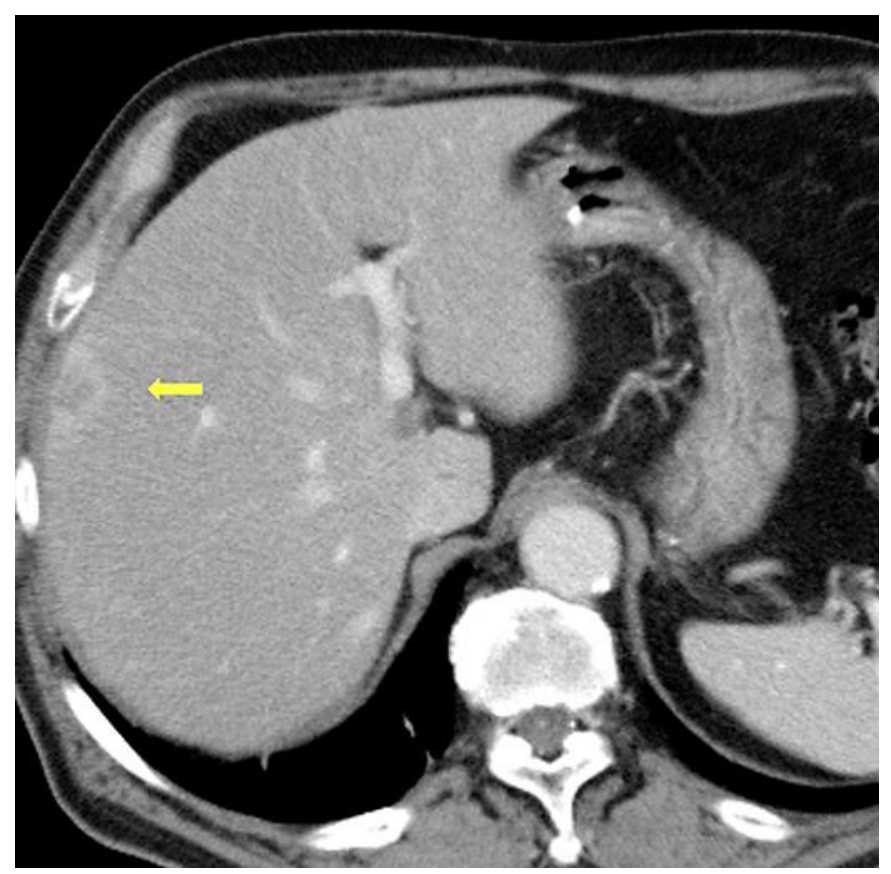

Fig. 2. Radiographic imaging. Computed tomography scan of the liver tumor of $3 \mathrm{~cm}$. The bulls eye symbol (arrow) in this lesion indicates a low-density area surrounded by a high-density capsule. 


\section{Case Reports in Gastroenterology}

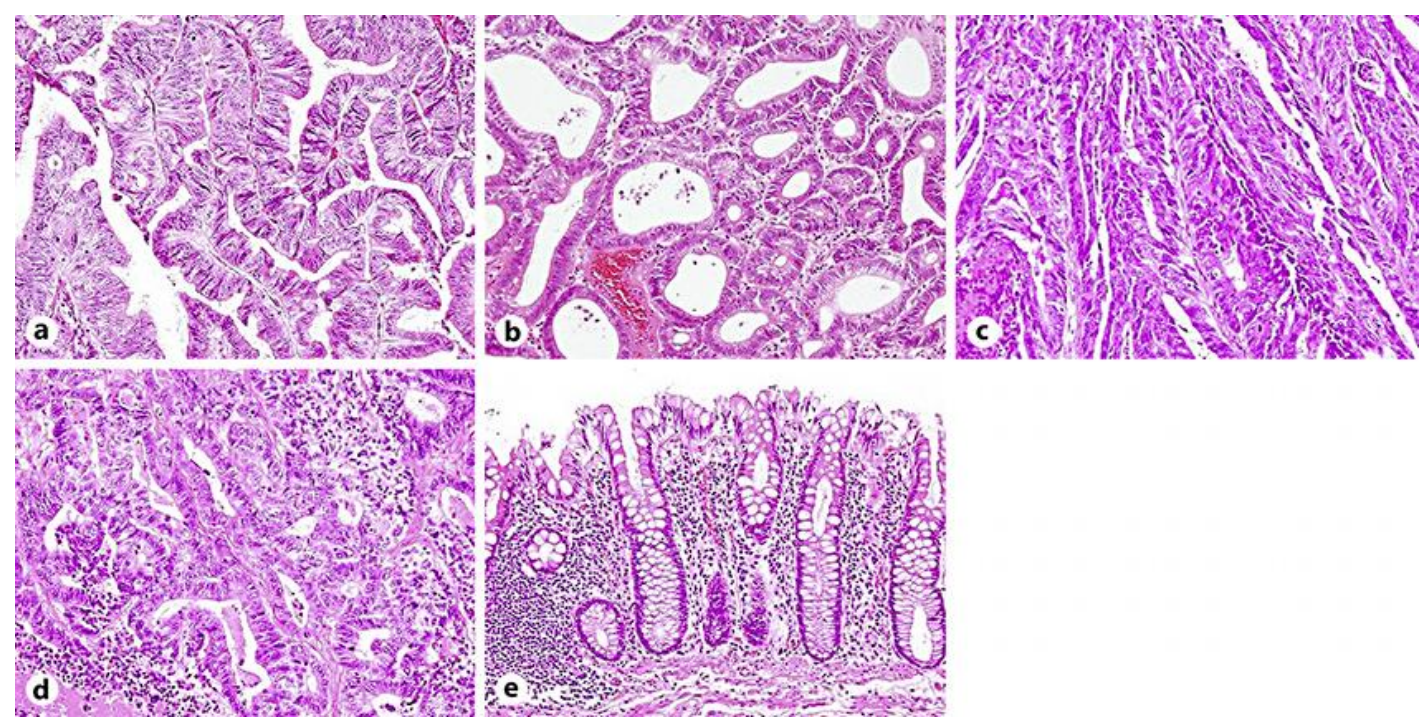

Fig. 3. Representative histopathological images of each lesion with hematoxylin-eosin staining. a Duodenal tumor with high-grade dysplasia glands $(\times 200)$. b Gastric tumor with high-grade dysplasia glands $(\times 200)$. c Rectal tumor with glands of adenocarcinoma $(\times 200)$. $\mathbf{d}$ Liver tumor with glands of adenocarcinoma similar to rectal adenocarcinoma $(\times 200)$. e Rectal normal mucosa analyzed as a control sample $(\times 100)$. 